\title{
Autism Diagnostics by 3D Texture Analysis of Cerebral White Matter Gyrifications
}

\author{
Ayman El-Baz ${ }^{1}$, Manuel F. Casanova ${ }^{2}$, Georgy Gimel'farb ${ }^{3}$, Meghan Mott $^{2}$, \\ and Andrew E. Switala ${ }^{2}$ \\ ${ }^{1}$ Bioengineering Department, University of Louisville, Louisville, KY, USA \\ ${ }^{2}$ Department of Psychiatry and Behavioral Science, University of Louisville, USA \\ ${ }^{3}$ Department of Computer Science, University of Auckland, New Zealand
}

\begin{abstract}
The importance of accurate early diagnostics of autism that severely affects personal behavior and communication skills cannot be overstated. Neuropathological studies have revealed an abnormal anatomy of the cerebral white matter (CWM) in autistic brains. We explore a possibility of distinguishing between autistic and normal brains by a quantitative shape analysis of CWM gyrifications on 3D proton density MRI (PD-MRI) images. Our approach consists of $(i)$ segmentation of the CWM on a 3D brain image using a deformable 3D boundary; (ii) extraction of gyrifications from the segmented CWM, and (iii) shape analysis to quantify thickness of the extracted gyrifications and classify autistic and normal subjects. The boundary evolution is controlled by two probabilistic models of visual appearance of 3D CWM: the learned prior and the current appearance model. Initial experimental results suggest that the proposed 3D texture analysis is a promising supplement to the current techniques for diagnosing autism.
\end{abstract}

\section{Introduction}

Autistic Spectrum Disorder (A.S.D.), or autism is a complex neurological disability that typically appears during the first three years of life and impacts development of social interaction and communication skills. Each individual is affected differently and at varying degrees, from milder forms in which intellectual ability is high but social interaction is low, to the most severe cases typified by unusual, self-injurious, and aggressive behaviors. The latter may persist throughout life and inflict a heavy burden on those who interact with autistic persons. Cognitive impairments may also last over time and often result in mental retardation in the majority of autistic individuals [1.

Neuropathological studies of autism have shown that children with autism have ordinary-size-brains at birth, but experience an acceleration of brain growth resulting, between two and four years of age, in increased brain volume relative to the normal (control) group 234. By adolescence and adulthood, differences in the mean brain size between the two groups diminish largely as a result of increased relative growth in the control group; nonetheless, there exists an abnormal anatomy of cerebral white matter (CWM) in autistic brains [34]. In 
addition, the deficits in the size of the corpus callosum and its sub-regions are well established in patients with autism relative to controls. To overcome limitations of the conventional volumetric-based diagnostics, we propose to quantitatively analyze shapes of CWM gyrifications considered as a texture on 3D PD-MRI images and use the found CWM abnormalities for robust classification of autistic vs. normal subjects. To the best of our knowledge, such an approach to automatically diagnose autism by 3D texture analysis of CWM gyrifications is the first of its kind.

Our objective is to quantify differences between the shape of CWM gyrifications for autistic and normal subjects. The proposed diagnostics is based on the three-step texture analysis of 3D PD-MRI brain images that is detalized in Sections 2 and 3. ( i) CWM segmentation from a 3D PD-MRI image using an evolving deformable boundary guided by probabilistic models of current and learned prior visual appearance of CWM; ( $i$ i) extraction of gyrifications from the segmented CSM, and ( $i i i)$ quantification of the thickness of the CSM gyrifications to perform classification. Experimental results and conclusions are given in Section 4 .

\section{Cerebral White Matter Segmentation}

Accurate CWM segmentation from a 3D PD-MRI image is a challenging problem because intensities in the CWM and surrounding organs are not clearly distinguishable. Thus, we segment the PD-MRI image using a conventional 3D parametric deformable boundary [6] but control its evolution with two original probabilistic models of visual appearance, namely, a learned CWM appearance prior accounting for translation- and rotation-invariant pairwise voxel interaction and a mixed model of voxel intensities in the current CWM and surrounding tissues.

The appearance prior is a Markov-Gibbs random field (MGRF) with multiple pairwise interaction having analytical identification (parameter estimation) from training data. The voxel-wise model of the current CSM appearance is extracted from the multi-modal mixed intensity distribution by its precise approximation with a linear combination of discrete Gaussians (LCDG) similar to the LCGapproximation in [7].

Let $(x, y, z)$ be Cartesian coordinates of 3D points. A finite 3D arithmetic lattice $\mathbf{R}=[(x, y, z): x=0, \ldots, X-1 ; y=0, \ldots, Y-1, z=1, \ldots, Z-1]$ supports a $3 \mathrm{D}$ image $g: \mathbf{R} \rightarrow \mathbf{Q}$ and its $3 \mathrm{D}$ region map $m: \mathbf{R} \rightarrow \mathbf{L}$ where $\mathbf{Q}=\{0,1, \ldots, Q-1\}$ and $\mathbf{L}=\{\mathrm{cwm}, \mathrm{bg}\}$ are finite sets of intensities and region labels, respectively. Each label, $m_{x, y, z}$, indicates whether a voxel $g_{x, y, z}$ in the corresponding intensity data set $g$ belongs to CWM, or background.

We use a conventional deformable boundary [6] that evolves in the direction minimizing its energy $E$ depending on internal, $\zeta_{\text {int }}(\mathbf{b})$, and external, $\zeta_{\text {ext }}(\mathbf{b})$, forces:

$$
E=E_{\text {int }}+E_{\text {ext }}=\int_{\mathbf{b}}\left(\zeta_{\text {int }}(\mathbf{b})+\zeta_{\text {ext }}(\mathbf{b})\right) d \mathbf{b}
$$

where $\mathbf{b}=\left[\mathbf{P}_{k}: k \in \mathbf{K}=\{1, \ldots, K\}\right]$ is a parametric surface with $K$ vertices $\mathbf{P}_{k}=\left(x_{k}, y_{k}, z_{k}\right)$. But we introduce a new type of the external energy involving 




Fig. 1. An initial (left) and normalized (right) PD-MRI image

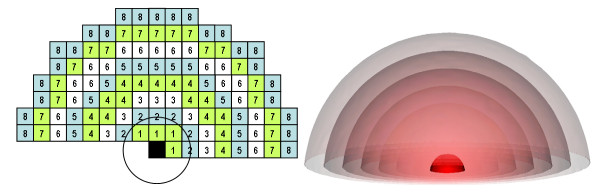

Fig. 2. Central-symmetric 2D (left) and 3D (right) neighborhoods for the eight distance ranges $\left[d_{\nu, \min }=\nu-0.5, d_{\nu, \max }=\right.$ $\nu+0.5) ; \nu \in \mathbf{N}=\{1, \ldots, 8\}$

the learned and on-going (current) visual appearance of CWM. Each image is normalized by mapping the signal range $\left[q_{\min }, q_{\max }\right]$ for each $3 \mathrm{D}$ data set to $[0,255]$ as in Fig. 1 in order to account for global contrast and offset deviations of intensities due to different sensors.

The normalized images are considered as samples of a prior MGRF model of the CWM appearance. To exclude any image alignment before segmentation, we use a generic translation- and rotation-invariant MGRF with only voxel-wise and central-symmetric pairwise voxel interaction. The latter is specified by a set $\mathbf{N}$ of characteristic central-symmetric voxel neighborhoods $\left\{\mathbf{n}_{\nu}: \nu \in \mathbf{N}\right\}$ on $\mathbf{R}$ and a corresponding set $\mathbf{V}$ of Gibbs potentials, one per neighborhood.

A central-symmetric voxel neighborhood $\mathbf{n}_{\nu}$ embraces all voxel pairs such that $(x, y, z)$-coordinate offsets between a voxel $(x, y, z)$ and its neighbor $\left(x^{\prime}, y^{\prime}, z^{\prime}\right)$ belong to an indexed semi-open interval $\left[d_{\nu, \min }, d_{\nu, \max }\right) ; \nu \in \mathbf{N} \subset\{1,2,3, \ldots\}$ of the inter-voxel distances: $d_{\nu, \text { min }} \leq \sqrt{\left(x-x^{\prime}\right)^{2}+\left(y-y^{\prime}\right)^{2}+\left(z-z^{\prime}\right)^{2}}<d_{\nu, \max }$. Figure 2 illustrates the neighborhoods $\mathbf{n}_{\nu}$ for the uniform distance ranges $[\nu-$ $0.5, \nu+0.5) ; \nu \in \mathbf{N}=\{1, \ldots, 8\}$.

Learning the appearance prior. Let $\mathbf{S}=\left\{\left(g_{t} . m_{t}\right): t=1, \ldots, T\right\}$ be a training set of $3 \mathrm{D}$ images with known region maps. Let $\mathbf{R}_{t}=\{(x, y, z):(x, y, z) \in$ $\left.\mathbf{R} \wedge m_{t ; x, y, z}=\mathrm{cwm}\right\}$ denote the part of $\mathbf{R}$ supporting CWM in the $t$-th training pair $\left(g_{t}, m_{t}\right) ; t=1, \ldots, T$. Let $\mathbf{C}_{\nu, t}$ be a family of voxel pairs in $\mathbf{R}_{t}^{2}$ with the co-ordinate offset $(\xi, \eta, \gamma) \in \mathbf{n}_{\nu}$ in a particular neighborhood. Let $\mathbf{F}_{\mathrm{vox}, t}$ and $\mathbf{F}_{\nu, t}$ be a joint empirical probability distribution of voxel intensities and of intensity co-occurrences, respectively, in the training CWM from $\mathbf{g}_{t}: \mathbf{F}_{\mathrm{vox}, t}=$ $\left[f_{\text {vox }, t}(q)=\frac{\left|\mathbf{R}_{t, q}\right|}{\left|\mathbf{R}_{t}\right|}: q \in \mathbf{Q}\right]$ and $\mathbf{F}_{\nu, t}=\left[f_{\nu, t}\left(q, q^{\prime}\right)=\frac{\left|\mathbf{C}_{\nu, t ; q, q^{\prime}}\right|}{\left|\mathbf{C}_{\nu, t}\right|}:\left(q, q^{\prime}\right) \in \mathbf{Q}^{2}\right]$ where $\mathbf{R}_{t, q}=\left\{(x, y, z):(x, y, z) \in \mathbf{R}_{t} \wedge g_{x, y, z}=q\right\}$ is a subset of voxels supporting the intensity $q$ and $\mathbf{C}_{\nu, t ; q, q^{\prime}}$ is a subset of the voxel pairs $\mathbf{c}_{\xi, \eta, \gamma}(x, y, z)=$ $((x, y, z),(x+\xi, y+\eta, z+\gamma)) \in \mathbf{R}_{t}^{2}$ supporting the intensity co-occurrence $\left(q, q^{\prime}\right)$ in the training CWM from $\mathbf{g}_{t}$. Let $\mathbf{V}_{\mathrm{vox}}=\left[V_{\mathrm{vox}}(q): q \in \mathbf{Q}\right]$ be a potential function of voxel intensities that describes the voxel-wise interaction. Let $\mathbf{V}_{\nu}=\left[V_{\nu}\left(q, q^{\prime}\right):\left(q, q^{\prime}\right) \in \mathbf{Q}^{2}\right]$ be a potential function of intensity co-occurrences in the neighboring voxel pairs that describes the pairwise interaction in the neighborhood $\mathbf{n}_{\nu} ; \nu \in \mathbf{N}$. 
The MGRF prior model of the $t$-th training pair is specified by the joint Gibbs probability distribution on the sublattice $\mathbf{R}_{t}$ :

$$
P_{t}=\frac{1}{Z_{t}} \exp \left(\left|\mathbf{R}_{t}\right|\left(\mathbf{V}_{\text {vox }}^{\top} \mathbf{F}_{\text {vox }, t}+\sum_{\nu \in \mathbf{N}} \rho_{\nu, t} \mathbf{V}_{\nu, t}^{\top} \mathbf{F}_{\nu, t}\right)\right)
$$

where $\rho_{\nu, t}=\left|\mathbf{C}_{\nu, t}\right| /\left|\mathbf{R}_{t}\right|$ is the average cardinality of $\mathbf{n}_{\nu}$ with respect to $\mathbf{R}_{t}$.

To simplify notation, let the CWM volumes in the training images be similar, so that $\left|\mathbf{R}_{t}\right| \approx R_{\mathrm{cwm}}$ and $\left|\mathbf{C}_{\nu, t}\right| \approx C_{\nu, \mathrm{cwm}}$ for $t=1, \ldots, T$. Here, $R_{\mathrm{cwm}}$ and $C_{\nu, \mathrm{cwm}}$ are the average cardinalities over the training set $\mathbf{S}=\left\{\left(g_{t} \cdot m_{t}\right): t=\right.$ $1, \ldots, T\}$. Assuming the independent samples, the joint probability distribution of intensities for all the training CWM images is as follows:

$$
P_{\mathbf{S}}=\frac{1}{Z} \exp \left(T R_{\mathrm{cwm}}\left(\mathbf{V}_{\mathrm{vox}}^{\top} \mathbf{F}_{\mathrm{vox}}+\sum_{\nu \in \mathbf{N}} \rho_{\nu} \mathbf{V}_{\nu}^{\top} \mathbf{F}_{\nu}\right)\right)
$$

where $\rho_{\nu}=C_{\nu, \mathrm{cwm}} / R_{\mathrm{cwm}}$, and the marginal empirical distributions of intensities $\mathbf{F}_{\text {vox }, \text { cwm }}$ and intensity co-occurrences $\mathbf{F}_{\nu, \mathrm{cwm}}$ describe now all the CWM images from the training set.

To identify the MGRF model described in Eq. 3, we have to estimate the Gibbs Potentials V. In this paper we introduce a new analytical maximum likelihood estimation for the Gibbs potentials (the mathematical proof for this new estimator is shown in our web sit 1 ).

$$
\begin{aligned}
& V_{\mathrm{vox}, \mathrm{cwm}}(q)=\log f_{\mathrm{vox}, \mathrm{cwm}}(q)-\frac{1}{Q} \sum_{\kappa \in \mathbf{Q}} \log f_{\mathrm{vox}, \mathrm{cwm}}(\kappa) ; \quad q \in \mathbf{Q} \\
& V_{\nu, \mathrm{cwm}}\left(q, q^{\prime}\right)=\lambda \rho_{\nu}\left(f_{\nu, \mathrm{cwm}}\left(q, q^{\prime}\right)-f_{\mathrm{vox}, \mathrm{cwm}}(q) f_{\mathrm{vox}, \mathrm{cwm}}\left(q^{\prime}\right)\right) ;\left(q, q^{\prime}\right) \in \mathbf{Q}^{2}
\end{aligned}
$$

where the common factor $\lambda$ is also computed analytically.

LCDG-models of Current appearance. Non-linear intensity variations in a data acquisition system due to a scanner type and scanning parameters affect visual appearance of CWM in each data set $\mathbf{g}$ to be segmented. Thus in addition to the learned appearance prior, we describe an on-going CWM appearance with a marginal intensity distribution within an evolving boundary $\mathbf{b}$ in $\mathbf{g}$. This distribution is considered as a dynamic mixture of two probability distributions that characterize the CWM and its background, respectively, and is partitioned into these two models using the EM-based approach in [7].

Boundary evolution under the two appearance models. We guide the boundary evolution in such a way that the following external energy term in Eq. (11) combining the learned prior and current appearance models within the boundary is minimized:

$$
\zeta_{\text {ext }}\left(\mathbf{P}_{k}=(x, y, z)\right)=-p_{\mathrm{vox}, \mathrm{cwm}}\left(g_{x, y, z}\right) \pi_{\mathbf{p}}\left(g_{x, y, z} \mid \mathbf{S}\right)
$$

where $p_{\text {vox ,cwm }}(q)$ is the marginal probability of the intensity $q$ in the above LCDG-model for the CWM and $\pi_{\mathbf{p}}(q \mid \mathbf{S})$ is the prior conditional probability

${ }^{1}$ http://louisville.edu/speed/bioengineering/faculty/bioengineering-full/ dr-ayman-el-baz/dr-ayman-el-bazs-lab.html 
of $q$, given the fixed current intensities in the characteristic central-symmetric neighborhood of $\mathbf{P}_{k}$ for the MGRF prior model of Eq. (3):

$$
\pi_{\mathbf{P}}\left(g_{x, y, z} \mid \mathbf{S}\right)=\frac{\exp \left(E_{\mathbf{P}}\left(g_{x, y, z} \mid \mathbf{S}\right)\right)}{\sum_{q \in \mathbf{Q}} \exp \left(E_{\mathbf{P}}(q \mid \mathbf{S})\right)}
$$

where $E_{\mathbf{P}}(q \mid \mathbf{S})$ is the conditional Gibbs energy of pairwise interaction for the voxel $\mathbf{P}$ provided that an intensity $q$ is assigned to it but the current intensities in all its neighbors over the characteristic neighborhoods $\mathbf{n}_{\nu} ; \nu \in \mathbf{N}$, remain fixed:

$$
E_{\mathbf{P}}(q \mid \mathbf{S})=V_{\text {vox }, \mathrm{cwm}}(q)+\sum_{\nu \in \mathbf{N}} \sum_{(\xi, \eta, \gamma) \in \mathbf{n}_{\nu}}\left(V_{\nu, \mathrm{cwm}}\left(g_{x-\xi, y-\eta, z-\gamma}, q\right)+V_{\nu, \mathrm{cwm}}\left(q, g_{x+\xi, y+\eta, z+\gamma}\right)\right)
$$

The evolution terminates after the total energy $E_{\mathbf{r}}$ of the $3 \mathrm{D}$ region $\mathbf{r} \subset \mathbf{R}$ inside the boundary $\mathbf{b}$ does not change:

$$
E_{\mathbf{r}}=\sum_{\forall \mathbf{P}=(x, y, z) \in \mathbf{r}} E_{\mathbf{P}}\left(g_{x, y, z} \mid \mathbf{S}\right)
$$

\section{Quantitative Analysis of CWM Gyrifications}

Our main hypothesis is that thickness of gyral CWM for normal subjects is greater than for autistic subjects as Fig. 3 suggests. To quantify this feature, we need first to automatically extract CWM gyrifications from the segmented CWM and then analyze their differences in order to classify normal and autistic persons.
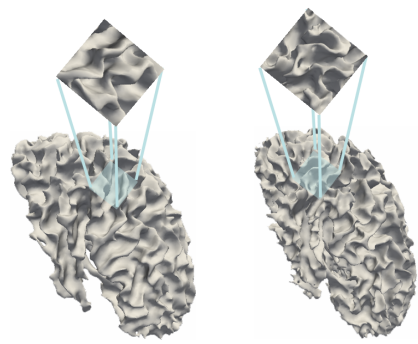
Fig. 3. Segmented CWM for a control tance map for the segmented CWM (the (left) and an autistic (right) patient. boundary found by segmentation is shown in Note that the CWM gyrifications the green) and the estimated class densities (right) autistic person appear thinner than for obtained from the mixed empirical distance the normal subject.
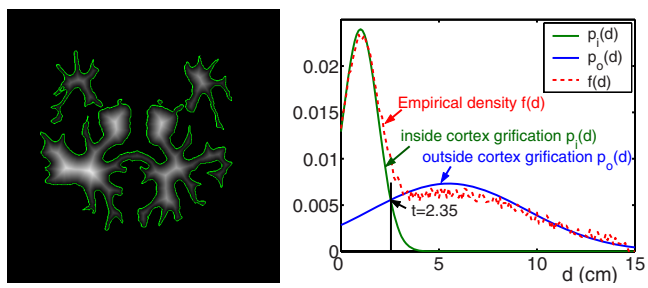

density for the segmented CWM

To extract gyrifications, we calculate the distance map inside the segmented 3D CWM by a fast marching level set method in [8]. The map gives the minimum Euclidean distance from each inner point of the segmented object to the object boundary (Fig. 4). Using the EM-based approach in 7], the mixed empirical marginal distribution of these distances is partitioned into two probability 

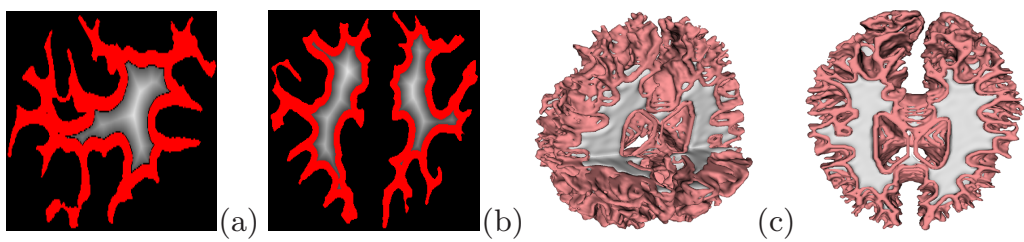

(d)

Fig. 5. 2D (a,b; red) and 3D (c, d; pink) visualization of the extracted CWM gyrifications

models: of the CWM gyrifications (class 1) and all other CWM tissues (class 2), respectively, shown in Fig. 4. Then the gyrifications are extracted using the optimum threshold that separates the two classes (see Fig. 51).

We propose to use the cumulative distribution function (CDF) of distances in a distance map inside the extracted CWM gyrifications as a generalized shape feature of the CWM structure. Figure [6] shows the CDFs for 14 subjects (7 autistic and 7 normal ones) selected for training. It is evident that the two classes, autistic and normal, are clearly separable using these CDFs.


Fig. 6. Cumulative distance distributions (a) inside the segmented training distance maps for 14(seven normal and seven autistic) subjects; the average CDFs for autistic and normal subjects (b), and the proposed classification (c,d) of unknown subjects (shown by green) by using the Levy distance $(\rho)$ to the average CDFs: (c) the normal and (d) the autistic subject

To classify the CDFs, the Levy distances between a CDF $F=\left[F_{u}: u=\right.$ $\left.0,1, \ldots, d_{\max }\right]$ in question for the distance map inside the extracted CWM gyrifications and the average CDFs $F_{A / N}$ in Fig. 6] serving as the templates of autistic or normal subjects are calculated [10] $: \rho\left(\mathbf{F}_{\mathbf{u}}, \mathbf{F}_{\mathbf{A} / \mathbf{N}}\right)=\min _{\alpha>0}\left\{\alpha: F_{A / N}(d-\alpha)-\right.$ $\left.\alpha \leq F_{u}(d) \leq F_{A / N}(d+\alpha)+\alpha\right\}$.

\section{Experimental Results and Conclusions}

The proposed approach has been tested on the 39 PD-MRI images of postmortem brains for 23 autistic patients (the mean interval between death and autopsy: 25.8 hours) and 16 controls (the mean interval between death and autopsy: 20.4 hours) obtained from the Autism Tissue Program (ATP). The brain tissues were scanned by a 1.5 Tesla GE MRI system with voxel resolution $0.3125 \times 0.3125 \times$ $1.6 \mathrm{~mm}^{3}$ using a proton density weighted imaging sequence protocol [5]). The 

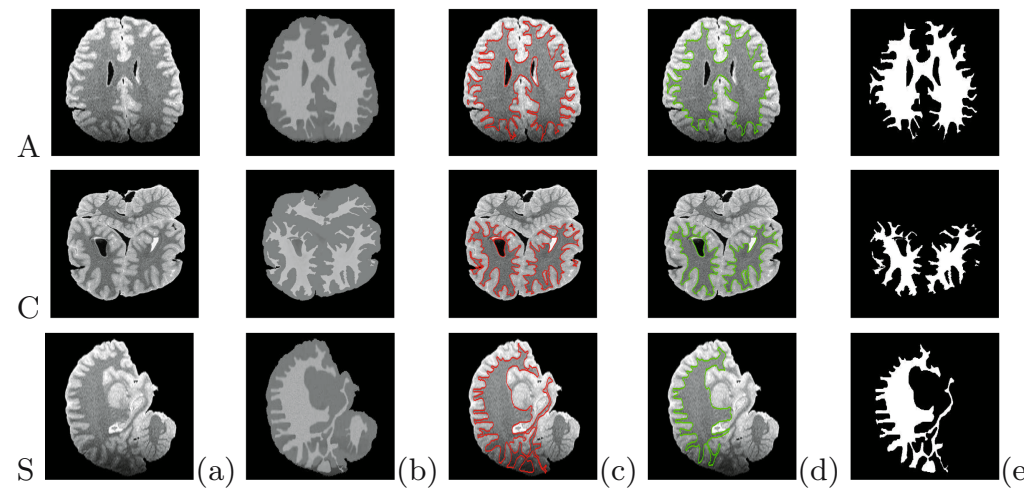

Fig. 7. Results of 3D CWM segmentation projected onto 2D axial (A), coronal (C), and saggital (S) planes for visualization: 2D profiles of the original PD-MRI images (a), pixel-wise Gibbs energies (b) for $\nu \leq 8$, our segmentation (c), the segmentation with the algorithm in [9], and (e) the radiologist's segmentation

"ground truth" diagnosis to evaluate the classification accuracy for each patient was given by the Autism Diagnostic Interview-Revised (ADIR).

Figure 7 demonstrates results of the CWM segmentation. The Gibbs energies for each CWM voxel are higher than for any other brain tissues. This is why the proposed approach is very accurate. The boundary evolution terminates after 226 iterations due to close to zero changes in the total energy. The error of our segmentation with respect to the radiologist's "ground truth" is $1.49 \%$. To highlight the advantages of our approach, we compare it to the most popular level-sets-based segmentation by Vese and Chan [9] where the level set evolution is controlled by region statistics, e.g. mean and variance. The segmentation error for their approach in the experiment in Fig. 7 is $9.6 \%$.

The main problem in the Vese and Chan's segmentation is that the errors usually occur just at the CWM gyrifications, which are the main features to discriminate between the autistic and normal subjects. The motivation behind our segmentation was to exclude such errors as far as possible.

The training subset for classification (14 persons shown in Fig. 6) was arbitrarily selected among all the the 39 subjects. The accuracy of classification of both the training and test subjects was evaluated using the $\chi^{2}$-test at the three confidence levels - 85\%,90\% and $95 \%$ - in order to examine significant differences in the Levy distances. As expected, the $85 \%$ confidence level yielded the best results - the correctly classified 22 out of 23 autistic subjects (a 96\% accuracy), and 15 out of 16 control subjects (a $94 \%$ accuracy). At the $90 \%$ confidence level, 22 out of 23 autistic subjects were still classified correctly, however, only 14 out of 16 control subjects were correct, bringing the accuracy rate for the control group down to $88 \%$. The $95 \%$ confidence level obviously gives the smaller accuracy rates for both the groups, namely, 20 out of 23 correct answers for autistic subjects $(87 \%)$ and still 14 out of 16 control subjects (88\%). Our preliminary 
explanation of the cases that are misclassified using the proposed approach is that there is a distortion in the geometry of the extracted cerebral white matter gyrifications. This distortion is due to fixation problems and removal of the brain from the skull because large deep cuts create distortions commonly revealed in MRI scans. The classification based on traditional volumetric approach is 11 out of 23 autistic subjects (a $48 \%$ accuracy), and 6 out of 16 control subjects (a $38 \%$ accuracy) at a 85 confidence interval, these results highlight the advantage of the proposed diagnostic approach. To show that the proposed approach is general and is not limited to ex-vivo data we tested the proposed approach on in-vivo data (Savant series [1]). The complete description of the Savant series and the classification results are shown in our web site2.

In total, these preliminary results show that the 3D texture analysis of the PD-MRI brain images is able to accurately discriminate between the autistic and normal subjects. Our proposal substantially differs from the known diagnosing techniques that exploit only volumetric descriptions of different brain structures and thus are in principle more sensitive to the selection of ages and segmentation errors 34. Contrastingly, the proposed approach derives efficient quantitative classification features from 3D shapes of different brain structures. Our experiments demonstrate statistically significant differences in the proposed generalized geometric characteristics of CWM gyrifications for 39 normal and autistic subjects under consideration.

In the future, we are going to investigate different brain structures in order to quantitatively characterize the development and changes of an autistic brain over time. Our investigation will not be limited to only the CWM but will also study the gray matter. Also, to validate and possibly modify the proposed approach, we will test it on larger data sets with known ground truth (doctors' diagnosis).

\section{References}

1. Minshew, N., Payton, J.: New perspectives in autism, part i. the clinical spectrum of autism. Curr. Probl. Pediatr. 18, 561-610 (1988)

2. Kanner, L.: Autistic disturbances of affective contact. Nervous Child 2, 250-250 (1943)

3. Aylward, E., Minshew, N., Field, K., Sparks, B., Singh, N.: Effects of age on brain volume and head circumference in autism. Neurology 59(2), 175-183 (2002)

4. Courchesne, R., Carper, R., Akshoomoff, N.: Evidence of brain overgrowth in the first year of life in autism. JAMA 290, 337-344 (2003)

5. Schumann, C., Buonocore, M., Amaral, D.: Magnetic resonance imaging of the post-mortem autistic brain. J. Autism. Dev. Disord. 31(6), 561-568 (2001)

6. Kass, M., Witkin, A., Terzopoulos, D.: Snakes: Active contour models. International Journal of Computer Vision 1, 321-331 (1987)

7. Farag, A., El-Baz, A., Gimel'farb, G.: Precise segmentation of multimodal images. IEEE Transactions on Image Processing 15(4), 952-968 (2006)

${ }^{2}$ http://louisville.edu/speed/bioengineering/faculty/bioengineering-full/dr-aymanel-baz/dr-ayman-el-bazs-lab.html 
8. Adalsteinsson, D., Sethian, J.: A fast level set method for propagating interfaces. Journal of Computational Physics 118, 269-277 (1995)

9. Vese, L., Chan, T.: A multiphase level set framework for image segmentation using the Mumford and Shah model. International Journal of Computer Vision 50(3), 271-293 (2002)

10. Lamperti, J.W.: Probability. J. Wiley \& Sons, New York (1996)

11. Hermelin, B.: Bright Splinters of the Mind, A Personal Study of Research with Autistic Savants. Jessica Kingsley Publishers Ltd (2001) 